\title{
The positive relationship between face identification and facial emotion perception ability extends to super-recognisers
}

L.M. Ali and J.P. Davis.

School of Human Sciences, University of Greenwich, London, SE10 9LS, UK.

Correspondence to Dr Josh P Davis (j.p.davis@gre.ac.uk)

ORCiD: https://orcid.org/0000-0003-0017-7159

Twitter: @GRecognisers

LinkedIn: https://www.linkedin.com/in/dr-josh-p-davis-7050137b/

Parts of this research were presented as a poster titled 'Super face recognisers have greater empathy and gregariousness', at PSYPAG (Psychology Postgraduate Affairs Group), Northumbria University, 26-28 July 2017.

A pre-print of the paper was uploaded to PsyArXiv for public view (DOI:

10.31234/osf.io/4ktrj)

The authors would like to thank Monika Durova, Diandra Bretfelean, Katy Weatherley, Nikolay Petrov, and Daisy Thomas for their help with participant recruitment and analyses.

This research was unfunded.

The authors declare no conflicts of interest for this work.

Data are available to download from DOI 10.17605/OSF.IO/8MBVR

Word count: 3621 


\begin{abstract}
Large individual differences in face identification ability and face emotion perception ability correlate in the neurotypical population. The current study recruited a large sample of participants aged 18-70-years $(n=687)$. The sample included superrecognisers with exceptional face identity processing skills $(n=74)$ to determine whether the relationship extends to the top of the ability spectrum. Participants completed one short-term face memory test, a simultaneous face matching test, and two face emotion perception tests. Face memory and face matching scores were moderately positively correlated with each other, and with total scores on the two face emotion perception tests. Super-recognisers outperformed typical-range ability controls at the perception of anger, fear, happy, and neutral, but not disgust or sadness. Identity processing and emotion perception accuracy across the whole sample was influenced by gender and age. From a theoretical perspective, these results demonstrate that correlations found between typical-range ability participants also extend to the top end of the face identification spectrum, implying that a common mechanism may drive performance. Practically, super-recognisers possessing superior face identity processing and emotion perception might enhance the performance of organisations such as police forces or security that rely on these attributes in their staff.
\end{abstract}

Keywords: Super-recognisers, face recognition, face matching, emotion perception, emotion recognition, individual differences 
Human face identification (e.g., Tardif et al., 2019; Verhallen, Bosten, Goodbourn, Lawrance-Owen, Bargary, \& Mollon, 2017) and face emotion perception abilities (e.g., Olderbak, Wilhelm, Hildebrandt, \& Quidbach, 2019) are crucial for social interactions. These skills positively correlate (e.g., Connolly, Young, \& Lewis, 2018; Rhodes et al., 2015), although ability at each varies substantially. Individuals unable to perceive specific face emotions have been identified (e.g., Calder, Young, Keane, \& Dean, 2000), while the face identification spectrum ranges from super-recognisers (SRs) with exceptional skills at unfamiliar face identification (e.g. Russell, Duchaine, \& Nakayama, 2009) to developmental prosopagnosics (DPs) who struggle to recognise close acquaintances (e.g. Bate \& Tree 2017).

The current study was the first to explore the intriguing theoretical possibility that super-recognition of identity may co-occur with superior face emotion perception. The research might also have applied impact. Some police forces have created specialist units to deploy police SRs (e.g., Davis, Lander, Evans, \& Jansari, 2016), and if SRs also possess superior emotion perception skills, such units might have wider-ranging benefits.

\section{Models of face identification and emotion perception}

Bruce and Young (1986; see also Haxby, Hoffman, \& Gobbini, 2000) proposed a functional face processing model with discrete components driving face identification and face emotion perception. Predicting no between face identification and emotion perception skills correlations, these models are supported by double dissociation cases in which some individuals are unable to process identity (prosopagnosia), but possess typical emotion perception ability (e.g., Bruyer, Latere, Serotti et al., 1983), and vice 
versa (e.g. Humphreys, Donnelly, \& Riddoch, 1993). However, when developing these models researchers assumed that unless deficits are observed, 'typical' humans possess roughly similar abilities. It has only recently been acknowledged that in the neurotypical population these skills vary far more widely than previously understood. It is therefore theoretically important to accommodate individual differences factors into face processing theories, and to ensure that correlations found in typical-ability participants, are found in SRs at the extreme of the spectrum (Young \& Noyes, 2019).

\section{Individual differences in face identification and emotion perception ability}

Individual differences in face identification ability appear mostly quantitative in nature (e.g. Tardif et al., 2019). However, dissociations between face memory and face perception (i.e. simultaneous face matching) tasks in some DPs (e.g. Bate \& Tree 2017) and SRs (e.g. Davis et al., 2016), suggest cross-domain independence (Megreya \& Burton, 2006). Nevertheless, an underlying face-specific cross-domain mechanism labelled $f$, explains about $25 \%$ of the variance in face identification ability (Verhallen et al., 2017).

Face identification and face emotion perception are both impacted by some of the same factors. Enhanced skills are associated with higher empathy levels (e.g., Baron-Cohen \& Wheelwright, 2004), and with being female (Bobak, Pampoulov, \& Bate, 2016; Olderbak et al., 2019; Susilo, Germine, \& Duchaine, 2013). They also bear a curvilinear relationship with age. Face identification ability peaks after about 30 years (e.g. Susilo et al., 2013), face emotion perception, between 15-30 years, while female's emotion perception advantage decreases with maturity (e.g., Olderbak et al., 2019; Rhodes et al., 2015). To further investigate these effects, the impact of age and gender 
on face identification and emotion perception ability were therefore also examined in the current study

\section{Face identification and face emotion perception ability}

Recent research has revealed correlations between face identification and face emotion perception ability. Rhodes et al. (2015) found that the face recognition test scores of 161 participants aged 17-30 years, correlated more strongly with emotion perception test scores than car recognition scores. Age positively correlated with both skills. No individual analyses were reported on the six emotions included in the test (anger, disgust, fear, happy, sadness, surprise). However, in a sample of 605 participants aged 18- to 87-years, Connelly et al. (2018) found that when general intelligence and short-term visual memory were controlled, scores on individual emotion perception tests (anger, disgust, fear, sadness, surprise, but not happy) positively correlated with face matching test scores. However, the authors reported no analyses evaluating age and emotion perception.

By recruiting a large participant pool, including many SRs, the current research aimed to extend these results by examining whether the relationship in the typical population extends to the top of the spectrum. We also tested as suggestion made by Connolly et al. (2018) that in comparison to correlations between face memory test scores and face emotion perception test scores, stronger correlations should be found between scores on face emotion perception and simultaneous face matching tests, as neither of the latter tasks draw on memory. However, the hallmark of super-recognition is superior face memory. Therefore, to test Connolly et al.'s hypothesis, participants completed one face matching (Glasgow Face Matching Test: Burton, White, \& McNeil, 
2010), and one face memory test (Cambridge Face Memory Test: Extended: CFMT+)

(Russell et al., 2009). The CFMT+ has commonly been used to allocate SRs, located in the approximately top $2 \%$ of the population to groups in previous research.

To measure emotion perception, the Face Emotion Recognition Test (Gur et al., 2002) assessed six emotions from full face photos (anger, disgust, fear, happy, neutral, sadness), while the Reading the Mind in the Eyes Test: Revised Version (Baron-Cohen, Wheelwright, Hill, Raste, \& Plumb, 2001) employs photos of the eyes region only. Neither test has been used previously to measure relationships with face identity test performances. However, if associations are robust, they should transfer to any test.

Face identification test scores and the perception of individual and total face emotion test scores were hypothesised to positively correlate, with SRs expected to outperform typical-ability controls. Furthermore, face identification ability, and face emotion perception ability, mature in a curvilinear manner, and similar ageing effects were expected here. Females were also expected to outperform males on all measures.

\section{Method}

\section{Participants}

Participants, attracted by media reports, had completed a Could you be a SuperRecogniser? Test on a University of Information withheld website. As recruitment rates could not be predicted, adult test finishers were invited to the current study for 5 weeks loaded on a Qualtrics survey system link. ${ }^{1}$ To ensure power to detect small correlation effect sizes, $r=0.20$, at $80 \%$ power and with $\alpha$ at $0.05, n=193$ was required. We were

\footnotetext{
${ }^{1}$ www.Qualtrics.com
} 
overpowered, as data of 687 participants who finished all tests were analysed allowing detection of very small effect sizes, $r=0.11$ [Gender: male $=189$, female $=495$, no response $=3$; Ethnicity: White-Caucasian $=545$ (79.3\%), British-Asian $=45(6.5 \%)$, other $=86(12.3 \%) ; 18-70$ years $(M=31.75, S D=11.22)$, missing $\left.=21^{2}\right]^{3}$.

Table 2 depicts SR group inclusion criteria on the CFMT+. These criteria matched those used in previous research (e.g. Satchell, Davis, Julle-Danière, Tupper, \& Marshman, 2019) for the top 2\% of the population (Bobak, Pampoulov et al., 2016). The recruitment method used tends to attract a high proportion of participants possessing far better face identification ability than is typical in the population. Similar effects were found here (see description of the CFMT+ in the materials section). The method used by Satchell et al. (2019) to control for this recruitment bias was employed, so that typical-range ability controls scored within 1 SD of the mean of Bobak, Pampoulov et al.'s (2016) participants (see Table 2). Ensuring SRs were exceptional face matchers, any scoring below SR's GFMT mean were excluded $(M=38.0)(n=29$ excluded out of 103), as were any controls outperforming SRs ( $n=61$ excluded out of 255).

Based on Satchell et al. (2019), we expected uneven group size recruitment, with a ratio of 1.4 in favour of controls. For power to detect medium (0.50) between-group effect sizes at $80 \%$ power a sample of $n=156$ was required. We recruited 74 SRs and 194 controls, allowing detection of small-to-medium (0.38) effect sizes.

\section{Materials}

\footnotetext{
${ }^{2}$ Between-gender mean age did not differ, $t(665)<1$.

${ }^{3}$ One additional participant scoring lowest on the CFMT+ (44 out of 102) was an extreme low outlier on the Face Emotion Test $(z=-7.07)$. As inclusion impacted conclusions their data were excluded.
} 
Cambridge Face Memory Test: Extended (CFMT+: for full description see Russell et al., 2009). In the first block of four, of this three-alternative choice 102-trial test, participants memorize photos of six Caucasian, male targets with external features removed (e.g. no hairstyle) and displayed from different viewpoints. The second and third blocks introduce additional distractors, visual noise, and faces displaying different expressions, while the fourth employs increasing visual noise, and repeating distractors.

Data on the CFMT+ were skewed [skewness $=-.904, \mathrm{SE}=.093$; Kurtosis $=.403, \mathrm{SE}$ $=.186$, Shapiro-Wilk, $\mathrm{W}(687)=.936, p<.001]$. With strong effect sizes, mean scores $(M=$ 83.7, $S D=11.0$ ) were significantly higher than Bobak, Pampoulov et al.'s (2016) representative UK sample, $\mathrm{t}(686)=31.09, p<.001$, Cohen's $\mathrm{d}=1.11$, indicative of a bias to recruit betterthan-typical participants.

Glasgow Face Matching Test (GFMT) (for full description see Burton et al., 2010). This 40-trial test displays pairs of full face male and female photos. With no time limits, participants decide if pairs are matched $(n=20)$ (i.e. two photos of the same person) or mismatched $(n=20)$ (i.e. of two different people who look alike).

Total scores on the test were skewed [skewness $=-1.066, \mathrm{SE}=.093 ;$ Kurtosis $=1.051$, $\mathrm{SE}=.186$, Shapiro-Wilk, $\mathrm{W}(687)=.911, \mathrm{p}<.001]$. With strong effect sizes, mean scores $(M=$ $36.2, S D=3.1)$ were significantly higher than Burton et al.'s (2010) normative sample $(M=$ $32.5, S D=2.43), t(686)=31.93, p<.001$, Cohen's $d=1.33$ again indicative of a recruitment bias.

Face Emotion Recognition Test (hereafter: 'Face Emotion Test') (for full description see Gur et al., 2002). This 48-trial test measures ability to match emotions from a list of six placed below each full-face photo (angry, disgusted, fearful, happy, 
neutral, sadness). Accuracy at correctly selecting each of the six emotions was calculated, as was total accuracy.

Total scores on the test were skewed $[$ skewness $=-1.061, \mathrm{SE}=.093 ;$ Kurtosis $=$ 2.093, $\mathrm{SE}=.186$, Shapiro-Wilk, $\mathrm{W}(687)=.938, p<.001]$. Total mean scores $(M=37.8, S D=$ 3.4) were significantly higher than Gur, Richard, et al.'s (2010) sample $(M=33.60, S D=3.10)$, $t(686)=32.81, p<.001$, Cohen's d $=1.29$.

Reading the Mind in the Eyes Test: Revised Version (hereafter: 'Eyes Emotion Test') (for full description see Baron-Cohen et al., 2001). This 36-trial test measures ability to decipher affective states from expressions in photos of the eyes only. Participants select one of four varying words describing a mental state or mood. Following precedent only total accuracy was analysed.

Scores on the test were skewed [skewness $=-.637, \mathrm{SE}=.093 ;$ Kurtosis $=.296, \mathrm{SE}=$ .186 , Shapiro-Wilk $\mathrm{W}(687)=.966, \mathrm{p}<.001]$. Mean total scores $(M=27.3, S D=3.8)$ were significantly higher than Baron-Cohen et al.'s (2001) sample $(M=26.20, S D=3.60), t(686)=$ $26.54, p<.001$, Cohen's $\mathrm{d}=.30$.

\section{Procedure}

After providing informed consent and demographic information, participants completed the CFMT+, the GFMT, then in a randomised order, the Face Emotion Test and the Eyes Emotion Test, before debriefing. ${ }^{4}$

\section{Results}

\footnotetext{
${ }^{4}$ Data from a face recognition ability self-belief question completed before the CFMT+ were not analysed as no face emotion perception question was included.
} 
Data can be downloaded from anonymised for review. IBM SPSS Statistics v25.0 was used for all analyses. Table 1 displays mean test scores and Pearson's correlation coefficients between scores, age and point-biserial gender correlations ( female $=1$, male $=0$ ).

Table 1 about here

As expected, CFMT + and GFMT scores were significantly, positively, and moderately correlated. Supporting hypotheses, face identity test scores significantly positively, but weakly correlated with both emotion perception test's total scores, and individual emotions except sadness (CFMT+, GFMT) and disgust (GFMT). Partly supporting predictions, coefficients between GFMT scores and both face emotion perception test's total scores were slightly stronger than those with the CFMT+.

Significant correlations showed that females outperformed males on the GFMT, and at fear and disgust perception only, albeit with small effect sizes. Age correlated significantly and negatively with accurate disgust, fear, and neutral emotion perception, but positively with sadness perception and Eyes Emotion Test total scores. Curvilinear regressions explore these contrasting age effects below.

Two multiple linear regression analyses were conducted with total scores on the two emotion tests as dependent variables. Predictors were age, gender $(1=$ female, $0=$ male), and CFMT+, and GFMT scores, as well as total scores on the alternative emotion perception test. Collinearity statistics were satisfactory on both the Face Emotion Test (tolerance $=.985-.777, \mathrm{VIF}=1.287-1.015)$, and the Eyes Emotion Test (tolerance $=$ $.987-.781, \mathrm{VIF}=1.281-1.018)$. 
A significant Face Emotion Test model, $\mathrm{R}^{2}$ adj $=0.137, F(5,661)=22.19, p<$ .001 , revealed that as expected given they measure the same construct, Eyes Emotion Test scores were the strongest predictor, $\beta=.226, t=6.026, p<.001$, while age (negatively), $\beta=-.139, t=-3.820, p<.001, \mathrm{CFMT}+, \beta=.165, t=4.099, p<.001$, and GFMT, $\beta=.101, t=2.467, p=.014$, scores (positively), were also significant. Gender were not significant $(p>.05)$.

A significant Eyes Emotion Test model, $\mathrm{R}^{2}$ adj $=.117, F(5,661)=18.57, p<$ .001 , revealed that only Face Emotion Test scores, $\beta=.231, t=6.026, p<.001$, age, $\beta=$ $.167, t=4.555, p<.001$, and GFMT, $\beta=.141, t=3.435, p .001$ test scores, all positively, were significant predictors. Gender and CFMT+ scores were not significant $(p>.2)$.

Age analyses: Participant age was skewed [skewness $=.976, \mathrm{SE}=.095$; Kurtosis $=.415, \mathrm{SE}=.189$, Shapiro-Wilk, $\mathrm{W}(666)=.916, \mathrm{p}<.001]$. Curvilinear analyses found age significantly predicted scores on the CFMT $+, r^{2}=.012, F(2,663)=$ $7.27, p=.001$, peak $\approx 38$-years; the GFMT, $r^{2}=.019, F(2,663)=7.44, p=.001$, peak $\approx$ 37 -years; the Face Emotion Test, $r^{2}=.016, F(2,663)=6.35, p=.002$, peak $\approx 28$-years; and the Eyes Emotion Test, $r^{2}=.031, F(2,664)=10.36, p<.001$, peak $\approx 46$-years. The lattter results explain the contrasting directional findings from the regressions described above.

Table 2 about here

Between-groups analyses: Table 2 displays independent-measures t-tests comparing groups. As expected, given recruitment criteria, with strong effect sizes, SRs significantly outperformed controls on the CFMT+, and the GFMT. With more 
moderate-to-small effect sizes, SRs also outperformed controls in terms of total scores on both emotion perception tests, as well as at the perception of all individual emotions except happy and sad.

\section{Discussion}

Replicating previous research (e.g. Connolly et al., 2018), face identification and face emotion perception test scores positively correlated. There were also weak significant positive correlations between face identification test scores and all Face Emotion Test individual emotions (angry, fear, happy, neutral), except sadness (CFMT+, GFMT) and disgust (GFMT). This was the first research to show that this relationship extends to the highest levels of ability. Super-recognisers (SRs) of face identity significantly outperformed typical-ability controls in terms of total scores on the Face Emotion Test and the Eyes Emotion Test, as well as at the perception of angry, disgust, fear, and neutral face emotions. No differences were found between controls and SRs at the perception of happy and sad emotions. The lack of effects with happy faces are probably due to accuracy being close-to-ceiling for both groups with this emotion. In contrast, the lowest rates of accuracy on any individual emotion were associated with sad perception. These results mostly match Connolly et al.'s (2018) research finding that the perception of five out of six emotions (anger, disgust, fearful, sadness, surprise, but not happy) positively correlated with face matching scores. Individual emotion outcome inconsistencies between research papers (i.e. see sadness perception), may simply be due to use of different stimuli, or emotion labelling difficulties. For instance, participants sometimes describe sad faces as tired, sleepy, dejected, and/or depressed (Ekman, 2003). 
Significant positive predictors of Face Emotion Test total scores (Gur et al., 2002) were Eyes Emotion Test scores (Baron-Cohen et al., 2001), the GFMT, and the CFMT+, while participant age was a negative predictor. In contrast, Face Emotion Test scores, GFMT, and age, but not CFMT+ scores, positively significantly predicted Eyes Emotion Test scores. The significant contribution of the GFMT, but not the CFMT+, in predicting Eyes Emotion scores may be due to similar requirements to use a finegrained feature-by-feature analytical approach to assess emotions from the eyes photos, and for simultaneous face matching tasks (see also Connelly et al., 2018). In contrast, full face availability on the Face Emotion Test may better link to the availability of full face cues in both the GFMT and the CFMT+. Further research employing alternative tests should investigate this proposal further. This would rule out effects being testspecific.

Previous research has provided evidence that an underlying mechanism $f$ drives individual differences in face identification ability (e.g. Verhallen et al., 2017). Connolly et al. (2018) suggested that from their findings of positive correlations between face emotion perception and simultaneous face matching, $f$ may also drive individual differences in emotion perception. The current results do not discount this. However, the far larger correlations between the CFMT+ and GFMT than between any other variables (see Table 1), also suggest $f$ may be a stronger driver of face identification ability than of emotion perception ability.

Although the authors did not report age-based analyses, Connolly et al.'s participants were also older than the current sample $(M=54.0 \mathrm{vs} .31 .7$ years $)$. Here, the positive curvilinear correlation between age and face identification test scores (peak age CFMT $+=38$-years, GFMT = 37-years), was slightly older than that found by Susilo et al. (2013) (peak age = 30-years). Intriguingly, the curvilinear relationship between age 
and emotion perception resulted in very different peak ages for the Face Emotion Test (28-years) and Eyes Emotion Test (46-years). Because of this, age negatively predicted overall scores on the Face Emotion Test, while positively predicting Eyes Emotion Test scores. The current results are correlational only, and it is not possible to claim that ability to perceive emotions from the eyes improves after perceiving emotions in full face images declines. Further research is required to investigate this relationship.

There were some limitations. As with other online research recruiting similar participant samples (e.g. Satchell et al., 2019), by more than one standard deviation, participants tended to be far superior at face identification (i.e. CFMT+ scores) than typical samples recruited to university laboratories (e.g. Bobak, Pompoulov et al., 2016). In addition, females substantially outnumbered males. This likely reflects a lack of interest in the topic from lower ability participants, particularly males, possessing self-awareness of their ability. Following precedent (e.g. Satchell et al., 2019), this recruitment bias was partly corrected for by comparing SRs with 'typical-ability' controls on all outcomes. For this, the control group was formed of those scoring within $1 \mathrm{SD}$ of the mean of a representative UK sample on the CFMT+ (see Bobak, Pampoulov et al., 2016). Despite the large participant sample ensuring sufficient power to detect weak effects, however, with a sample displaying a restricted ability range, correlational strengths were likely to have been reduced, potentially obscuring significant effects. This reduced ability range may also explain the weak gender-related effects. Females normally outperform males at face emotion perception (e.g., Olderbak et al., 2019). Here, females outperformed males on the GFMT, and at fear and disgust perception only, albeit with weak effect sizes. There were no gender effects associated with the CFMT+ or either emotion test total scores. 
Future research could recruit a more gender-balanced sample, as well as evaluate whether the relationship between identity and emotion perception extends to the lowest extremes of ability (i.e. in developmental prosopagnosics). Additional focus should also investigate correlations between face identification ability and individual emotion perception using different test batteries. It is theoretically important to investigate whether superior face identification skills are associated with the perception of all face emotions, or only with a subset as revealed here.

In conclusion, the outcomes of the current research were consistent with past findings revealing positive correlations between face identity and face emotion perception abilities. The study was also the first to demonstrate that the correlations in these skills found in typical-range ability participants, extend to super-recognisers at the top of the face identification ability spectrum.

\section{References}

Baron-Cohen, S., \& Wheelwright, S. (2004). The empathy quotient: An investigation of adults with Asperger syndrome or High Functioning Autism, and normal sex differences. Journal of Autism and Developmental Disorders, 34, 163-175. doi:10.1023/B\%3AJADD.0000022607.19833.00

Baron-Cohen, S., Wheelwright, S., Hill, J., Raste, Y., \& Plumb, I. (2001). The Reading the Mind in the Eyes Test revised version: a study with normal adults and adults with Asperger Syndrome or high functioning autism. Journal of Child Psychiatry and Psychiatry, 42, 241-252. doi:10.1111/1469-7610.00715 
Face and emotion recognition

Bate, S., \& Tree, J. J. (2017). The definition and diagnosis of developmental prosopagnosia. The Quarterly Journal of Experimental Psychology, 70, 193200. doi:10.1080/17470218.2016.11955414

Benjamini, Y., \& Hochberg, Y. (1995). Controlling the false discovery rate: A practical and powerful approach to multiple testing. Journal of the Royal Statistical Society. Series B (Methodological), 57(1), 289-300. www.jstor.org/stable/2346101

Bobak, A. K., Pampoulov, P., \& Bate, S. (2016). Detecting superior face recognition skills in a large sample of young British adults. Frontiers in Psychology, 7, 1-18. doi:10.3389/fpsyg.2016.01378

Bruce, V., \& Young, A. (1986). Understanding face recognition. British Journal of Psychology, 77, 305-327. doi:10.1111/j.2044-8295.1986.tb02199.x

Bruyer, R., Latere, C., Serotti, X., Fayereisen, P., Strypstein, E., Pierrard E., et al. (1983). A case of prosopagnosia with some preserved overt remembrance of familiar faces. Brain and Cognition, 2, 257-284.

Burton, M., White, D., \& McNeill A. (2010). The Glasgow face matching test. Behavior Research Methods, 42, 286-291. doi:10.3758/BRM.42.1.286

Calder, A. J., Young, A. W., Keane, J., \& Dean, M. (2000). Configural information in facial expression perception. Journal of Experimental Psychological Perceptual Performance, 2, 527-551. doi:10.1037/0096-1523.26.2.527

Connolly, H. L., Young, A. W., \& Lewis, G. J. (2018). Recognition of facial expression and identity in part reflects a common ability, independent of general intelligence and visual short-term memory. Cognition and Emotion, 33(6), 11191128. doi:10.1080/02699931.2018.1535425 
Davis, J. P., Lander, K., Evans, R., \& Jansari, A. (2016). Investigating predictors of superior face recognition ability in police super-recognisers. Applied Cognitive Psychology, 30(6), 827-840. DOI: 10.1002/acp.3260

Ekman, P. (2003). Emotions revealed: Recognizing faces and feelings to improve communication and emotional life. New York City, NY: Henry Holt \& Co.

Gur, R. C., Richard, J., Hughett, P., Calkins, M. E., Macy, L., Bilker, W. B., Brensinger, C., \& Gur, R. E. (2010). A cognitive neuroscience based computerized battery for efficient measurement of individual differences: Standardization and initial construct validation. Journal of Neuroscience Methods, 187(2), 254-262. doi:10.1016/j.jneumeth.2009.11.017

Gur R. C., Sara, R., Hagendoorn, M., Marom, O., Hughett, P., Macy, L., Turner, T., Bajcsy, R., Posner, A., \& Gur, R.E. (2002). A method for obtaining 3dimensional facial expressions and its standardization for use in neurocognitive studies. Journal of Neuroscience Methods, 115, 137-143.

Haxby, J. V., Hoffman, E. A., \& Gobbini, M. I. (2000). The distributed human neural system for face perception. Trends in Cognitive Sciences, 4(6), 223-233. doi:10.1016/S1364-6613(00)01482-0

Humphreys, G. W., Donnelly, N., \& Riddoch, M. J. (1993). Expression is computed separately for moving and static faces: neuropsychological evidence. Neuropsychologia, 31(2), 173 -181. doi:10.1016/0028-3932(93)90045-2

Megreya, A. M., \& Burton, A. M. (2006). Unfamiliar faces are not faces: Evidence from a matching task. Memory and Cognition, 34(4), 865-876. doi:10.3758/BF03193433 
Olderbak, S., Wilhelm, O., Hildebrandt, A., \& Quidbach, J. (2018). Sex differences in facial emotion perception ability across the lifespan. Cognition and Emotion. doi: org/10.1080/02699931.2018.1454403

Rhodes, G., Pond, S., Burton, N., Kloth, N., Jeffery, L., Bell, J., Erwing, L., Calder, A.J., \& Palermo, R. (2015). How distinct is the coding of face identity and expression? Evidence for some common dimensions in face space. Cognition, 142, 123-137. doi:10.1016/j.cognition.2015.05.012

Russell, R., Duchaine, B., \& Nakayama, K. (2009). Super-recognisers: people with extraordinary face recognition ability. Psychonomic Bulletin \& Review, 16(2), 252-257. doi:10.3758/PBR.16.2.252

Satchell, L., Davis, J. P., Julle-Danière, E., Tupper, N., \& Marshman, P. (2019). Recognising faces but not traits: Accurate personality judgment from faces is unrelated to superior face memory. Journal of Research in Personality. doi:10.31234/osf.io/63whf

Susilo, T., Germine, L., \& Duchaine, B. (2013). Face recognition ability matures late: Evidence from individual differences in young adults. Journal of Experimental Psychology: Human Perception and Performance, 39(5), 1212-1217. doi:10.1037/a0033469

Tardiff, J., Morin Duchesne, X., Cohan, S., Royer, J., Blais, C., Fiset, D., Duchaine, B., \& Gosselin, F. (2019). Use of face information varies systematically from developmental prosopagnosics to super-recognizers. Psychological Science, 30(2), 300-308. doi: 10.1177/0956797618811338

Verhallen, R. J., Bosten, J. M., Goodbourn, P. T., Lawrance-Owen, A. J., Bargary, G. \& Mollon, J.D. (2017). General and specific factors in the processing of faces. Vision Research, 141, 217-227. doi:10.1016/j.visres.2016.12.014 
Face and emotion recognition

Young, A.W., \& Noyes, E. (2019). We need to talk about super-recognizers: Invited commentary on: Ramon, M., Bobak, A. K., \& White, D. Super-recognizers:

From the lab to the world and back again. British Journal of Psychology, 110(3), 492-494. doi:10.1111/bjop.12395 
Table 1. Pearson's correlations between scores on the face identification, emotion perception tests, and age and gender $(n=687)$.

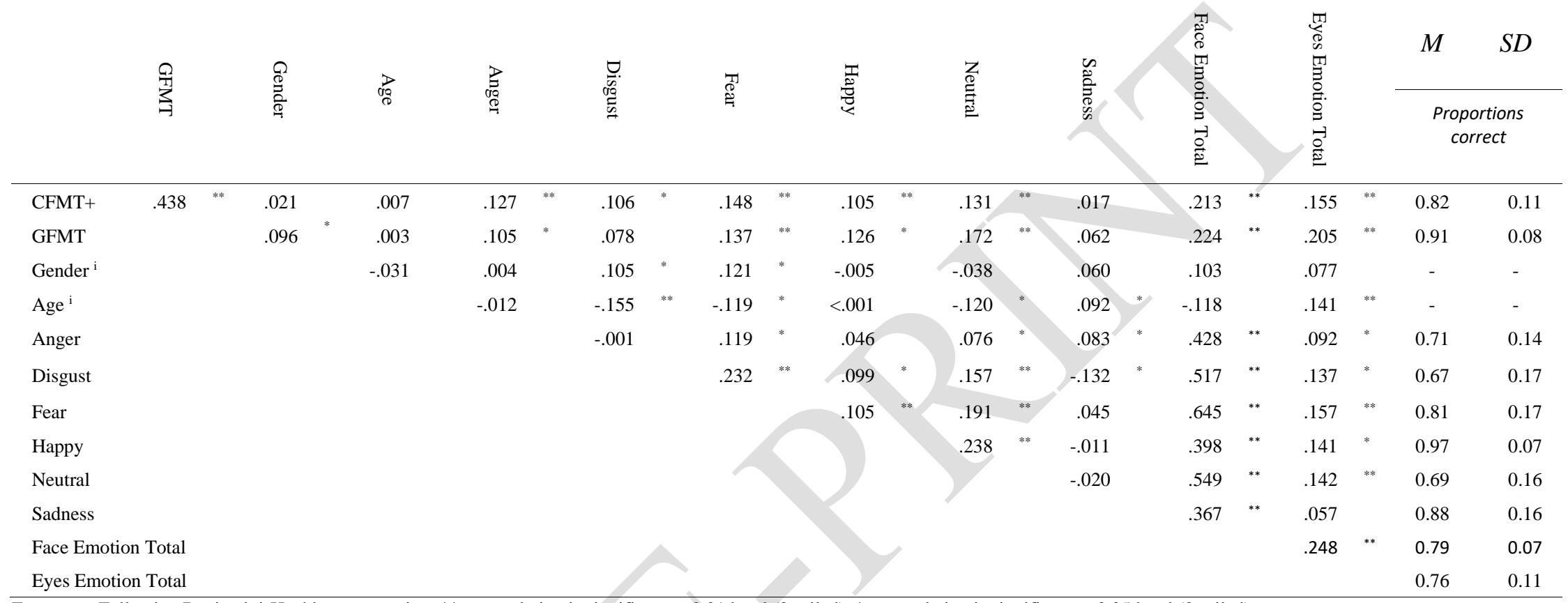

Footnotes: Following Benjamini-Hochberg correction, $* *=$ correlation is significant at 0.01 level (2-tailed), $*=$ correlation is significant at 0.05 level $(2$-tailed).

Spearman's correlations conducted on the same table revealed virtually identical results

i Data were missing (age: $n=21$, gender: $n=3$ ). 
Table 2: SR and typical-ability range recruitment criteria, demographics and results of tests comparing SRs with controls. Emboldened p's indicate significance following the BenjaminiHochberg (1995) procedure.

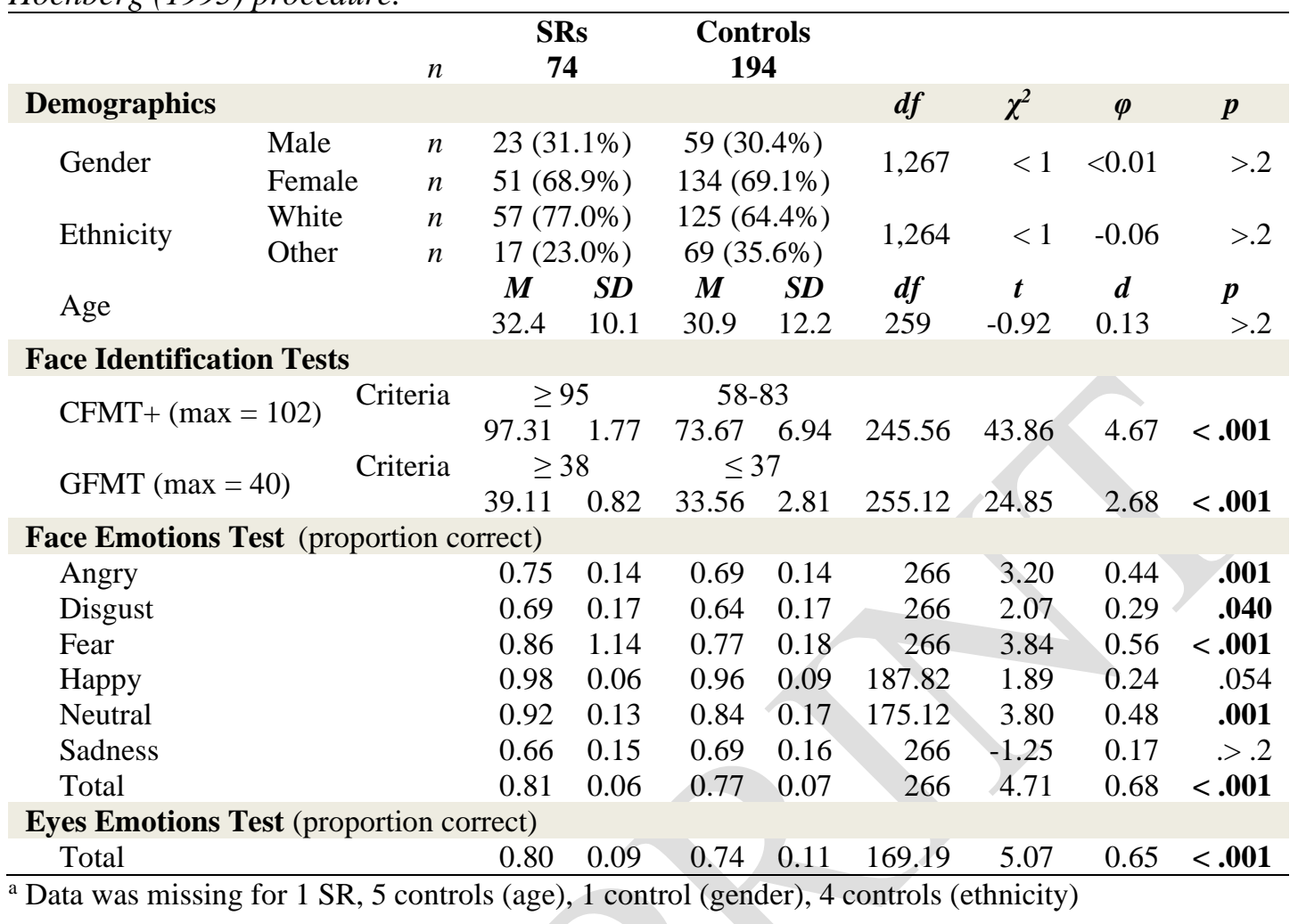

